\title{
LA SIMULIIDOSE HUMAINE EN FRANCE : SON ANCIENNETÉ, SA RÉPARTITION, LES ESPÈCES ANTHROPOPHILES
}

\author{
J. C. BEAUCOURNU**, F. BEAUCOURNU-SAGUEZ**, S. CHEVRIER*
}

RÉSUMÉ

Le problème humain de la Simuliidose est, pour la France, envisagé en soulignant la croissance brutale et semblant relativement récente des cas signalés. Les zones et les espèces de simulies anthro-pophiles sont données.

Summary: The human Simuliidosis in France: antiquity, repartition, anthropophilic species.

The problem of the human Simuliidosis (caused by black flies) is reviewed for the France: important and, perhaps, relatively recent increasure from the known human attacks by black flies. Countries and anthropophilic species are cited.
Si le terme de Simuliidose est récent, ce qu'il recouvre, c'est-à-dire la nuisance non vectorielle due aux Simulies est sans doute aussi ancien, en ce qui nous concerne, que la cohabitation de l'Homme avec ces «moucherons bossus ». La pollution, souvent et à juste titre incriminée car entraînant la pullulation de certaines espèces, n'a fait qu'augmenter les cas et changer les espèces en cause; elle doit de plus être limitée : Simulium ornatum existait par millions jusqu'aux années 40 aux environs de Londres; elle a aujourd'hui disparu (Crosskey, 1985). Les modifications de l'environnement, évidentes en Afrique noire pour ce qui concerne l'irrigation, n'ont pas été évaluées dans notre pays. Dans l'Ouest de la France a contrario, le remembrement entraînant la disparition des ruisseaux temporaires et un drainage forcené des autres, aurait plutôt un effet nocif sur la faune en faisant disparaître, entre autres, diverses espèces de simulies non anthropophiles d'ailleurs, comme Greniera fabrei (Beaucournu-Saguez, non publié).

Nous nous contenterons d'évoquer le problème de l'impact médical en éliminant les aspects cliniques et physiopathologiques qui nous entraîneraient à de trop longues discussions; puis nous verrons, uniquement pour la France, l'ancienneté de ce phénomène, sa répartition dans le temps et l'espace, les espèces agressives.

* Laboratoire de Parasitologie médicale, Faculté de Médecine, avenue du Professeur Léon-Bernard, F 35043 Rennes Cedex.

** Laboratoire de Parasitologie pharmaceutique, UFR Pharmacie, même adresse.

Accepté le: 5 septembre 1992.

\section{LE PROBLÈME MÉDICAL}

Les références anciennes à leur agressivité hors de nos contrées abondent. Pour le Canada, par exemple, Davies et coll. (1962) ont fait une revue bibliographique de citations s'étalant de 1604 à nos jours. Crosskey (1990) donne une compilation de textes recouvrant pratiquement toutes les régions concernées. Nous citons simplement le récit suivant qui semble avoir échappé à ces auteurs. Bach en 1863 , explorant le grand nord canadien rapporte : «Parmi les nombreuses misères inhérentes à la vie aventureuse du voyageur, il n'en est point de plus insupportable et de plus humiliante que la torture que vous fait subir cette peste ailée. En vain vous essayez de vous défendre contre ces petits buveurs de sang, en vain en abattez-vous des milliers, d'autres milliers arrivent aussitôt pour venger la mort de leurs compagnons, et vous ne tardez pas à vous convaincre que vous avez engagé un combat où votre défaite est certaine... » et il ajoute quelques pages plus loin « Comment décrire les souffrances que nous causèrent, dans ce trajet, les moustiques et leurs alliés les maringouins? Nos figures ruisselaient de sang comme si l'on y eût appliqué des sangsues. La cuisante et irritante douleur que nous éprouvions, immédiatement suivie d'inflammation et de vertige, nous rendait presque fous... ».

Cette nuisance, due à de multiples espèces, est particulièrement connue de toutes les Amériques (sauf le sud de la zone néotropicale), de la zone paléarctique (des îles britanniques au Japon, mais pas la sous-région méditerranéenne), des Iles Marquises, de la Nouvelle-Zélande, ... 
Nous éliminons ici la zone afrotropicale où l'impact vectoriel domine.

Il est difficile de ne pas évoquer la mouche de Goloubatz du Danube (Simulium columbaschense) : c'est à son propos, et sans entrer ici dans le détail des milliers d'animaux domestiques qui en furent victimes, que Schönbauer en 1795 mentionne les premiers cas mortels connus chez l'Homme.

Bien que les décès soient rarissimes, après lui Riley en 1887 en note dans la basse vallée du Mississipi (mais Crosskey (1990) se demande s'il n'y a pas eu confusion avec des morsures de crotales!). Cependant pour l'Amérique du Nord, Johannsen (1903), Williston (1908), Forbes (1912), ... et d'autres admettent cette mortalité. Alcock (1920) en signale à son tour dans la région himalayenne.

La clinique est riche et variée généralement axée sur la douleur, l'œdème, les lésions hémorragiques. Nous n'en parlerons ici que pour noter quelques entités nosologiques :

- la « Fièvre à simulies» qui est connue de l'État de New York; Crosskey en rapproche des syndromes observés au Japon et en Angleterre depuis 1969 où, dans ce dernier pays, ils sont provoqués par la "Mouche de Blandford" (Simulium posticatum);

- Pinheiro et coll., en 1974, décrivent du Brésil amazonien le "Syndrome hémorragique d'Altamira ", marqué en particulier par une forte thrombopénie pouvant être fatale : ces auteurs pensent pouvoir le rattacher aux piqûres de simulies ( $S$. ? amazonicum, Simulium sp.);

- Inter alia, citons Diaz et coll. (1989), qui également au Brésil, incriminent ces insectes (en l'occurrence $S$. pruinosum) dans le déclenchement de Pemphigus (Pemphigus foliacé endémique) par exacerbation de phénomènes autoimmuns.

Rappelons, pour tomber dans l'anecdote, qu'Émile Brumpt en 1909 était persuadé du rôle des simulies dans la transmission de la lèpre.

Pour clore ce survol rappelons, une fois encore après Crosskey mais ceci est épidémiologiquement fondamental, que le fait d'avoir des simulies tournant autour de soi ne correspond le plus souvent à aucun signe d'agressivité. Tous ceux qui, par exemple font de la randonnée en montagne le savent, bien que Grenier $(1947,1953)$ s'y soit laissé prendre. L'observation de Peterson (1977) en Islande est caractéristique : " Jamais, écrit-il, je ne vis tant de simulies en même temps. Elles furent toutes sur nous grouillant dans toutes les ouvertures imaginables de notre habillement et de nos personnes. Elles recouvraient mes lunettes n'empêchant de voir au travers. Il devenait difficile de respirer et j'ai le souvenir particulier du bourdonnement ténu de ces millions d'ailes minuscules. J'ai fait tourbillonner mon filet à insectes devant moi afin de pouvoir respirer et voir dans la mesure du possible. En dépit de cela, ni mon compagnon, ni moi ne furent piqués, même une seule fois ".
Personnellement, nous avons été des dizaines de fois, dans le Queyras (Hautes-Alpes) en particulier, entre 2500 et $3000 \mathrm{~m}$, escortés par des essaims tourbillonnants de Prosimulium: exceptionnellement un ou deux individus se posaient, mais là encore, jamais aucun de nous ne fut piqué... malgré nos souhaits!

\section{AnCIEnNeté et RÉPARtition EN FranCe}

Il s'agit évidemment d'une notion fragile, correspondant peut-être surtout à une prise de conscience du problème par une fin de $\mathrm{xIX}^{\mathrm{e}}$ siècle éprise de progrès et avide de science. L'existence de noms vernaculaires en divers points de France (mouchottes dans l'Est (Beurey et coll., 1983), guipets (M. et Mme Martin, inédit) ou guipettes ( $D^{r}$ Gielfrich, inédit) en Ille-et-Vilaine, ...) pourrait indiquer leur intérêt et une certaine pérennité du phénomène. Mais il convient toutefois d'être prudent car ces appellations désignent divers "moucherons", hématophages ou non et quelquefois n'appartenant même pas aux diptères, comme ces essaims estivaux de petits staphylinides. Les arabics (sic) signalés par de Rivière en 1826 de Camargue, nom que Rolland (1911) attribue aux Simulies sont très vraisemblablement, ici, des Leptoconops. Dans le Vaucluse, par contre, les arabis sont manifestement les simulies. Des noms «de circonstance» peuvent également les désigner en les reliant à un biotope (mouche des oliviers, Grouiller et coll., 1984), à une saison (moucheron de printemps, Rousset et coll., 1983) ou à une plante qui peut-être leur fournit le nectar indispensable (mouche des vignes, Grouiller et coll., 1984; mouche du colza, Auct., non publié).

Quoi qu'il en soit, la première mention nous paraissant valable semble pour la France être celle de Tisserant en 1863 (carte 1) dans le canton de Condrieu, département du Rhône : des hordes de simulies (le mot est lâché!) identifiées comme S. maculatum. attaquent les troupeaux, entraînant une mortalité. Dans son rapport, Tisserant établit clairement les préférences trophiques:

- animaux domestiques : « solipèdes et bêtes à cornes » essentiellement...,

- conducteurs d'attelages, vulgum pecus!

- et aussi l'HOMME « J'ai eu, écrit « le Professeur Tisserant ", ... mille peines de m'en débarrasser et d'éviter leurs piqûres qui sont excessivement douloureuses... ». Seuls les premiers cités, les animaux domestiques, ont eu des décès!

Sur des arguments sans aucune valeur scientifique, Mégnin (1875) corrigera l'identification donnée par Tisserant et attribuera l'épizootie de Condrieu à $S$. cinereum (Macquart, 1834): S. cinereum est un synonyme de S. equinum (Linné, 1758). 

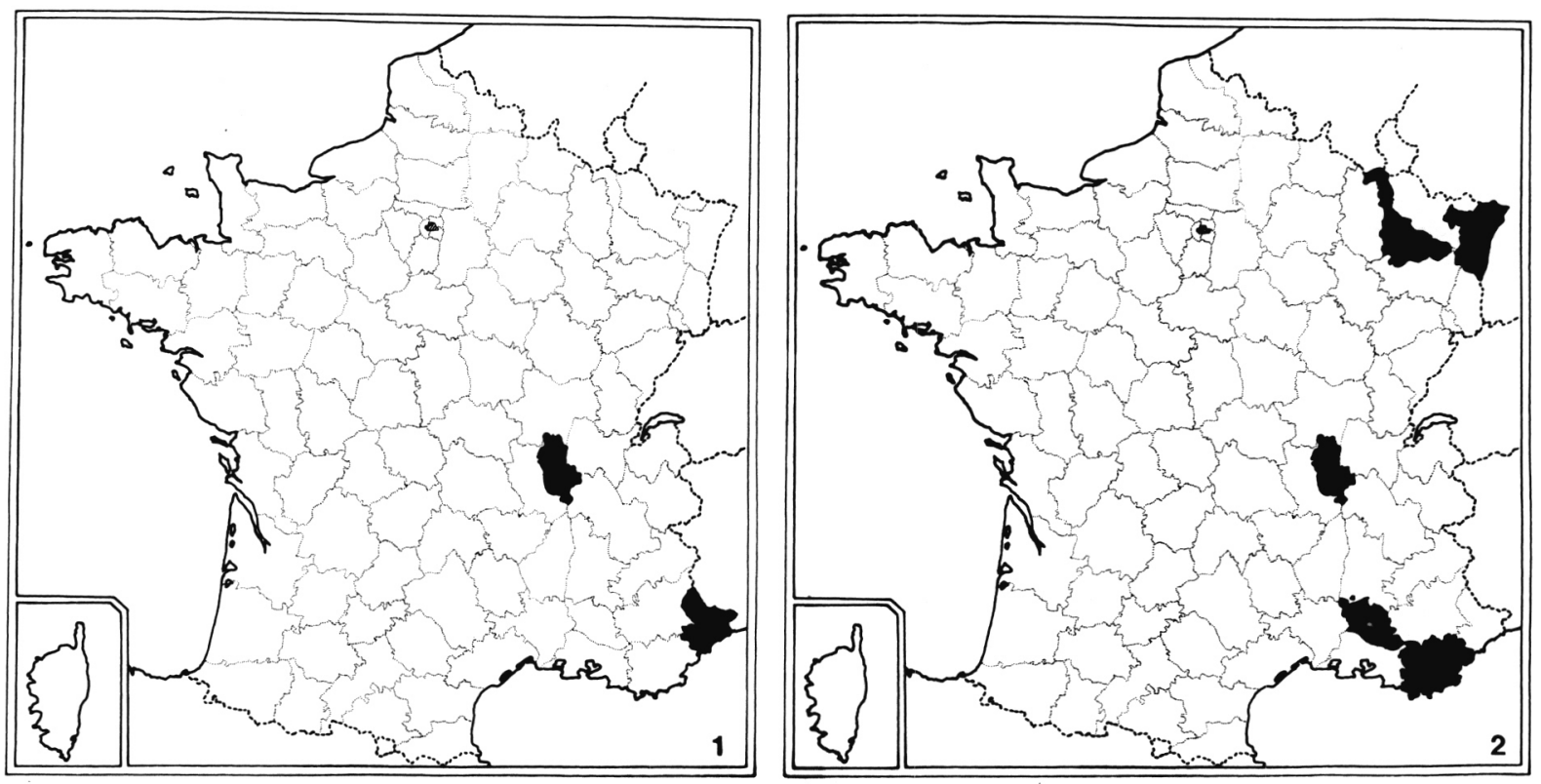

Cartes. - 1: Cas ou foyers humains répertoriés de 1863 à 1945 (83 ans); 2 : Cas ou foyers humains répertoriés de 1946 à 1977 (32 ans).

TABleAu I. - Espèces de Simulies signalées en France comme anthropophiles de 1863 à 1992.

Bases de l'identification

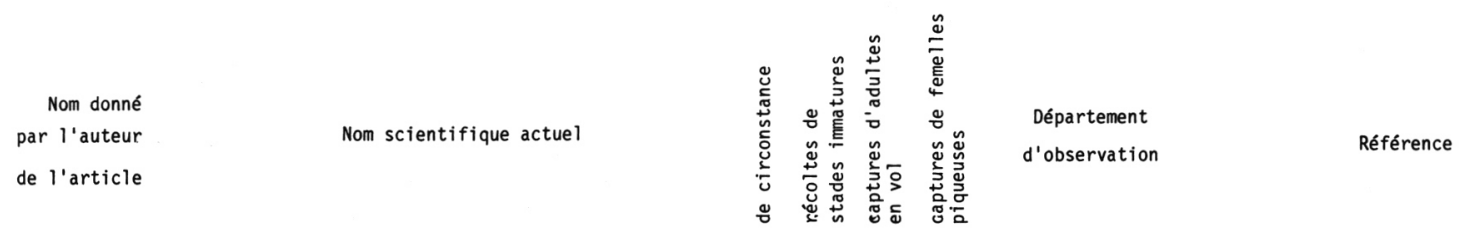

\begin{tabular}{|c|c|c|c|c|c|c|c|}
\hline Simulium ornatum & S. (Simulium) ornatum Meigen, 1818 (complexe) & $x$ & & & & Meurthe-et-Moselle & Michon et Lienhart, 1946 \\
\hline S. ornatum & $d^{\circ}$ & & $x$ & & $x$ & Bas-Rhin & Callot, 1952 \\
\hline Odagmia gr. ornatum? & $d^{\circ}$ & & & & $x ?$ & Vaucluse & Joubert et Pravé, 1972 \\
\hline S. ornatum? & $d^{\circ}$ & & $x$ & & & Yonne & Rousset et coll., 1983 \\
\hline S. ornatum & $d^{\circ}$ & & $x$ & & & Marne & $\begin{array}{l}\text { Lavaud et coll.. } 1991 \\
\text { (et autres travaux sur même gîte) }\end{array}$ \\
\hline S. ornatum & $d^{\circ}$ & & $x$ & & $x$ & Vosges & Jacques, 1984 \\
\hline S. ornatum & $d^{\circ}$ & & $x$ & & $x$ & Ardennes & Beaucournu-Saguez, 1980 : non publié \\
\hline S. ornatum & $d^{\circ}$ & & $x$ & & & Yvelines & $d^{\circ} \quad, 1985:$ non publié \\
\hline S. ornatum (complexe) & $d^{\circ}$ & & $x$ & & $x$ & Vaucluse & Beaucournu-Saguez et coll.., en préparation \\
\hline S. Bezzii & S. (Tetisimulium) bezzii (Corti, 1914) & & & & $x$ & Var & Brunhes et Philippon, 1966 \\
\hline Tetisimulium bezzii ? & $d^{\circ}$ & & & & $x ?$ & Vaucluse & Joubert et Pravé, 1972 \\
\hline S. bezzii & $d^{\circ}$ & & $x$ & & $x$ & $d^{\circ}$ & Beaucournu-Saguez et coll., en préparation \\
\hline $\begin{array}{l}\text { S. erythrocephalum } \\
\text { var. sericatum }\end{array}$ & S. (Boophthora) erythrocephalum (De Geer, 1776) & & & & $x$ & Bas-Rhin & Callot, 1951 \\
\hline S. erythrocephalum & $d^{\circ}$ & & $x$ & & $x$ & Vaucluse & Beaucournu-Saguez et coll.,1992, sous presse \\
\hline S. erythrocephalum & $d^{\circ}$ & & & & $x$ & Gard & $d^{\circ} \quad$. 1992, non publ ié \\
\hline S. posticatum & S. (S.) posticatum Meigen, 1838 & & $x$ & $x$ & $x$ & Eure & $\begin{array}{l}\text { Beaucournu-Saguez et coll., } 1990 \\
+ \text { Chevrier, } 1990\end{array}$ \\
\hline S. posticatum & $d^{\circ}$ & & $x$ & $x$ & $x$ & Ille-et-Vilaine & Beaucournu-Saguez, 1991 : non publié \\
\hline S. cinereum & S. (Wilhelmia) equinum (Linné, 1758) & $x$ & & & & Rhône & $\begin{array}{l}\text { Mégnin, } 1875 \\
\text { (? émendation de Tisserant, 1863) }\end{array}$ \\
\hline Wilhelmia equina? & $d^{\circ}$ & & $x$ & & & Yonne & Rousset et coll., 1983 \\
\hline S. reptans & S. (S.) reptans (Linné, 1758) & & & & $x$ & Rhône & Grenier, 1953 \\
\hline S. reptans & $d^{\circ}$ & & $x$ & & & Marne & Lavaud et coll., 1991 (et autres travaux) \\
\hline S. variegatum & S. (S.) variegatum Meigen, 1818 & & & & $x$ & Var & Brunhes et Philippon, 1966 \\
\hline S. variegatum & $d^{\circ}$ & & & & $x$ & Alpes-Maritimes & Beaucournu-Saguez, 1992 : non publié \\
\hline S. latipes & S. (Hellichiella) latipes (Meigen, 1804) & & $x$ & & & Bas-Rhin & Callot, 1951 \\
\hline S. maculatum & S. (Byssodon) maculatum (Meigen, 1804) & & & $x ?$ & $x ?$ & Rhône & Tisserant, 1863 (err. det.?,cf. texte) \\
\hline S. morsitans & S. (S.) morsitans Edwards, 1915 & & & & $x$ & Bas-Rhin & Callot, 1951 \\
\hline
\end{tabular}


Presque contemporaine est l'observation rapportée par Zurcher et Margollé (1864) et nous ne nous y attarderons que pour montrer, inter alia, la naissance d'une ... contrevérité. «... des ouvriers employés au reboisement d'une partie de la montagne de l'Espérou (dans les Cévennes, au début de septembre) ont été témoins d'un phénomène extraordinaire dans ces contrées. A deux heures du soir, un bruit sourd et monotone, à peu près analogue à celui que produit un orage lointain, fixa leur attention sur un épais brouillard qui traversait un mamelon à environ deux kilomètres devant eux. L'air était très calme... ils ne furent pas peu surpris lorsque, s'étant avancés, ils reconnurent que c'était une colonne immense de moucherons dont la longueur était de plus de $1500 \mathrm{~m}$ sur une largeur de 30 et une hauteur de $50 \ldots$ ». Ce texte est repris par Girard (1868) sans commentaire mais il le place dans le souschapitre des némocères (nématocères). Railliet (1895) parle de nuées de moustiques noirâtres, termes qu'il emprunte à une autre phrase de Girard, et cite cette « observation de Girard » au chapitre des Simulies. Phisalix (1922), recopie Railliet en remplaçant seulement les mots moucherons noirâtres par simulies. Grenier (1953) revient en l'abrégeant au texte de Girard, qu'il authentifie en l'incluant, sans discussion aucune, dans le chapitre "Biologie des imagos " de sa Faune des Simuliidae. Voici donc une observation aussi difficile à contester (puisque tout le monde acquiesce)... qu'à entériner.

Par la suite ce sont seulement des attaques de chevaux, en 1869 et en 1875 (Mégnin, 1875), en 1877 (Mégnin, 1895), de chevreuils, en 1909 (Bouvier, cité par Phisalix, 1922) que nous retrouvons : toutes ces observations ont lieu dans le quart nord-est de la France : St-Mihiel (Meuse), Charenton (Val-de-Marne), Fontainebleau et Melun (Seine-etMarne). Ce n'était pas encore l'époque où l'on dérangerait le médecin pour un fait sans doute estimé banal; nous n'évoquerons plus désormais les cas concernant les vétérinaires, sauf lorsque l'homme y sera impliqué.

Toutefois, à la même époque (1908), Marchoux et Bourret, partisans de la transmission vectorielle de la lèpre, dans une enquête sur un petit foyer situé dans la vallée de Valdeblore (Alpes-Maritimes), notent « on n'y trouve que deux espèces d'insectes parasites, des puces et aussi, au printemps et en été, des diptères très répandus dans les pays à lèpre, des simulies ». Si la notion précise d'agression par ces insectes n'est pas donnée, nous pensons qu'on peut la déduire de l'argumentation des auteurs.

Un grand silence, ou une lacune dans nos recherches, va s'étendre jusqu'en 1946 (carte 2) où Michon et Lienhart attirent l'attention des médecins sur les piqûres de simulies dans la région de Nancy (Meurthe-et-Moselle). En 1951 et 1952, Callot donne plusieurs sites du Bas-Rhin où il fut piqué par diverses simulies d'espèces différentes. Grenier (1953) dans sa Monographie note qu' « en France, on possède peu d'informations précises sur l'agressivité des
Simuliidae, cependant certains faits laissent penser que cette question ne doit pas être complètement perdue de vue... " et comme donnée inédite il ne cite qu'une observation de Roman « dans la région de Lyon... piquant l'homme occasionnellement $»$.

En $1966^{1}$, Brunhes et Philippon dans un travail faunistique signalent qu'ils furent piqués dans le Var par deux espèces et notent « des femelles de Prosimulium inflatum nous ont assaillis avec insistance en pleine journée dans la vallée de Casterine (Alpes-Maritimes)... mais sans toutefois aller jusqu'à nous piquer ». Nous retrouvons ici la notion impérative que nous devons faire établir par nos patients ou nos correspondants entre simulies « assaillantes », en fait tourbillonnantes, et simulies piqueuses!

Joubert et Pravé (1972) étudiant l'épidémiologie de la myxomatose, signalent incidemment qu'ils sont piqués par des simulies appartenant à une ou deux espèces. Ceci se passe en Haute-Provence, dans le Vaucluse, département que nous retrouverons plus loin. Le Docteur Gilot a bien voulu nous préciser qu'il s'agissait de la commune de Malaucène.

En 1979 et 1981, Noirtin et coll. étudient, sous deux angles voisins, un assez grave foyer de simuliidose bovine dans les Vosges. Quelques cas de piqûres de l'homme (Jacques, 1984) se rencontrent à cette occasion. Soit « déclic » diagnostique, soit augmentation du phénomène, les observations vont maintenant se succéder (carte 3 ).

En 1983, Beurey et coll. signalent un cas se répétant chaque année concernant une femme de Meurthe-et-Moselle; la même année (et également en 1985, où le même cas est repris plus à fond), Rousset et coll. décrivent une autre attaque isolée (dans l'Yonne), mais une enquête leur permet de savoir que les habitants de la région sont régulièrement piqués. Grouiller et coll. (1984) donnent une intéressante note sur les piqûres de Simuliidae dans le Var (et peut-être les Bouches-du-Rhône). Il semble là s'agir d'un « foyer permanent ». En 1985 c'est Bourgeois qui, pour le Jura, donne l'histoire d'un patient piqué chaque année de 1979 à 1984 mais signale également qu'il ne s'agit pas d'un cas isolé.

Beaucournu-Saguez et coll. (1990), Chevrier (1990), étudient un foyer, petit en surface mais important par son impact médical, situé dans l'Eure : c'est le premier connu en France de la "Mouche de Blandford " (S. posticatum) évoquée au début de cette note. La même année et en 1991, paraissent plusieurs travaux (Caron, 1990; Gérardin, 1990; Prévoteau, 1990; Bouchet, 1991; Lavaud et coll., 1991, ce dernier faisant, sous l'angle qui nous occupe, la synthèse des autres) sur une série de petits foyers de la Marne : peut-être moins explosifs que celui de l'Eure, leur appari-

1. Nous éliminerons la note de BAzEx et coll. (1958) qui n'est pas géographiquement localisée et n'apporte aucun élément permettant de la ranger dans les observations de Simuliidose; quant à celle de Verdaguer et coll. (1985), c'est une revue intéressante mais schématisée du problème sans apports nouveaux. 

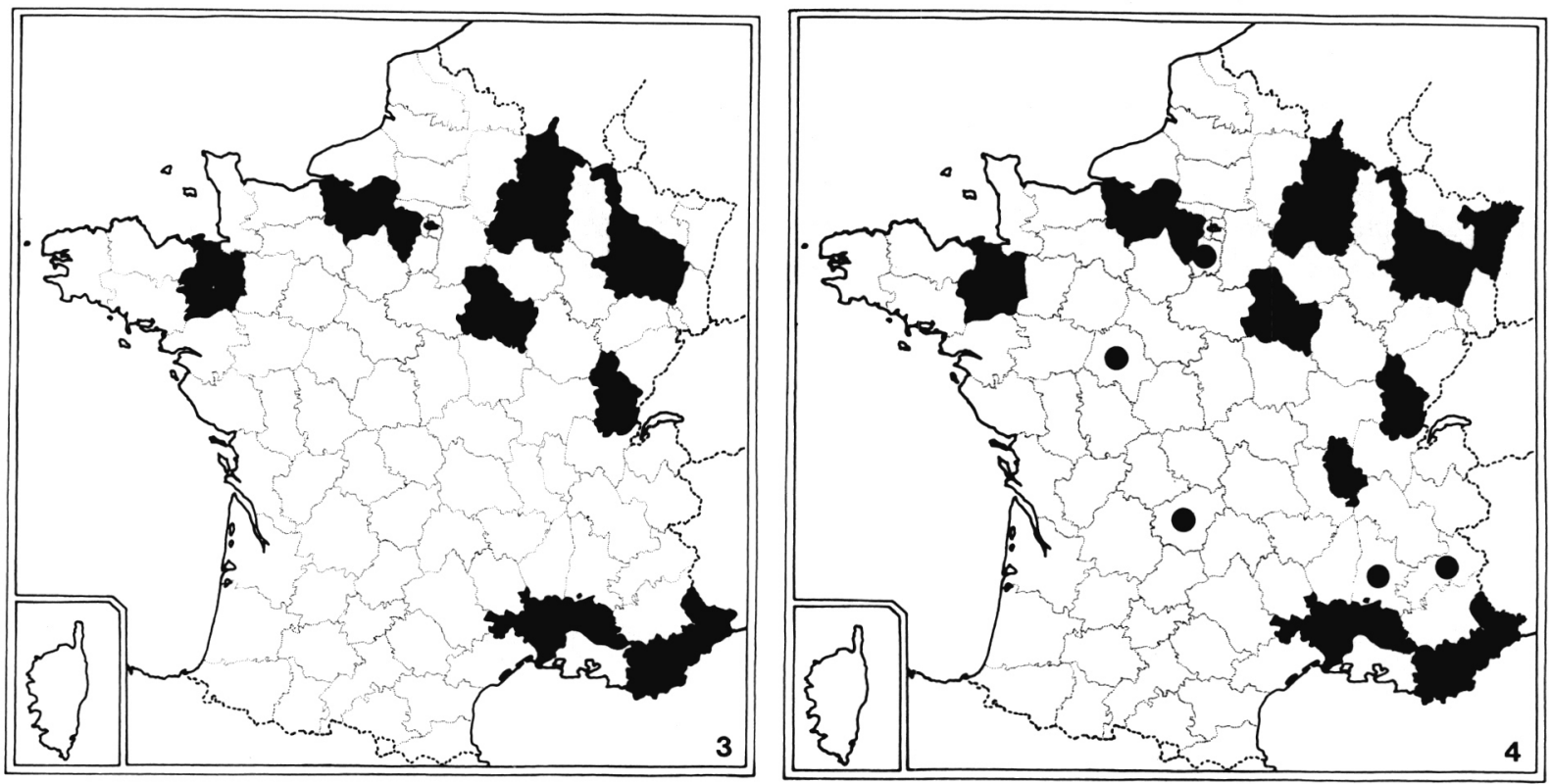

Cartes. - 3: Cas ou foyers humains répertoriés de 1978 à 1992 (15 ans); 4 : Carte récapitulative (le cercle noir indique que, dans le département concerné, la simulie ne fut pas spécifiquement identifiée).

tion est un peu plus ancienne puisque semblant remonter à 1986, alors que celui de Damville (Eure) « naît » en 1987.

Dernier en date en tant que tel, le foyer du Vaucluse (signalé par Meller-Melloul sous le nom d'Arabies (sic) en 1991 et faisant l'objet d'une note préliminaire par Beaucournu-Saguez et coll. en 1992) s'avère particulièrement intéressant car au moins ponctuellement ancien et " alimenté » par trois espèces anthropophiles. Il est très actif puisque le médecin d'une des petites communes concernées (petite mais très touristique) évalue à 500 le nombre de consultations annuelles.

En dehors de ces publications (et nous ne pouvons espérer être exhaustifs, trop d'observations intéressantes paraissant dans des revues difficiles à consulter) nous avons connaissance de cas isolés, ou le plus souvent de petits foyers, dans les Ardennes, les Yvelines, l'Essonne, l'Indre-et-Loire, la Corrèze, la Drôme, les Hautes-Alpes, le Gard et nous confirmons l'ancienne publication de Marchoux et Bourret (1908) sur les Alpes-Maritimes en y citant trois zones d'agression : St-Martin-Vesubie ( $D^{\mathrm{r}}$ Gaud), St-Paul-deVence ( $\mathrm{D}^{\mathrm{r}}$ Clastrier) et Breil-sur-Roya ( $\mathrm{D}^{\mathrm{r}}$ Deramond). Enfin, en Ille-et-Vilaine, nous connaissons une patiente (et une seule!) régulièrement piquée à St-Senoux depuis 4 ans (carte 4).

\section{Les Simulies ANTHRopophiles}

Crosskey (1990) estime à 1550 le nombre de représentants actuellement connus de cette Famille. Il est difficile de donner le chiffre exact du nombre de simulies de la faune française. Cette difficulté vient bien sûr de l'insuffisance des prospections, mais surtout de la taxonomie très difficile de cette famille où beaucoup d' " espèces " sont des complexes, ou ont donné lieu à des synonymies très abondantes (Zwick et Crosskey, 1981; Crosskey, in Kim et Merritt, 1986; Crosskey, 1991; ...) qui font que la même espèce peut apparaître sous plus d'une dizaine de noms! Pour la France continentale, on peut estimer à moins de 40 les taxa recensés. Sur ce nombre, étant bien entendu que cela ne leur enlèvera en rien leur étiquette " zoophile " et plus exactement "mammalophile", combien sont à retenir comme anthropophiles?

Les articles ou observations énumérés ci-dessus font état de 12 taxa différents (tableau I). Compte tenu de la taxonomie actuelle, ce nombre peut être abaissé à 9 , la mention de $S$. latipes devant désigner un autre membre du groupe "ruficorne " et deux noms étant mis en synonymie (S. cinereum et $S$. erythrocephalum var. sericatum).

Si l'on ne retient que les espèces impliquées sans aucune ambiguité dans des cas ou des foyers de simuliidose humaine, l'identité étant alors basée sur la femelle piqueuse, ce chiffre tombe à cinq :

- S. ornatum (complexe) (carte 5), la plus souvent citée et, sinon ubiquiste, du moins largement répandue comme espèce agressive,

- S. posticatum (carte 6), piqueuse dans deux zones seulement mais à l'origine du foyer de Damville, dans l'Eure,

- S. variegatum, encore mal connue sous l'angle qui nous occupe, seulement "inculpée » dans le Sud-Est, 

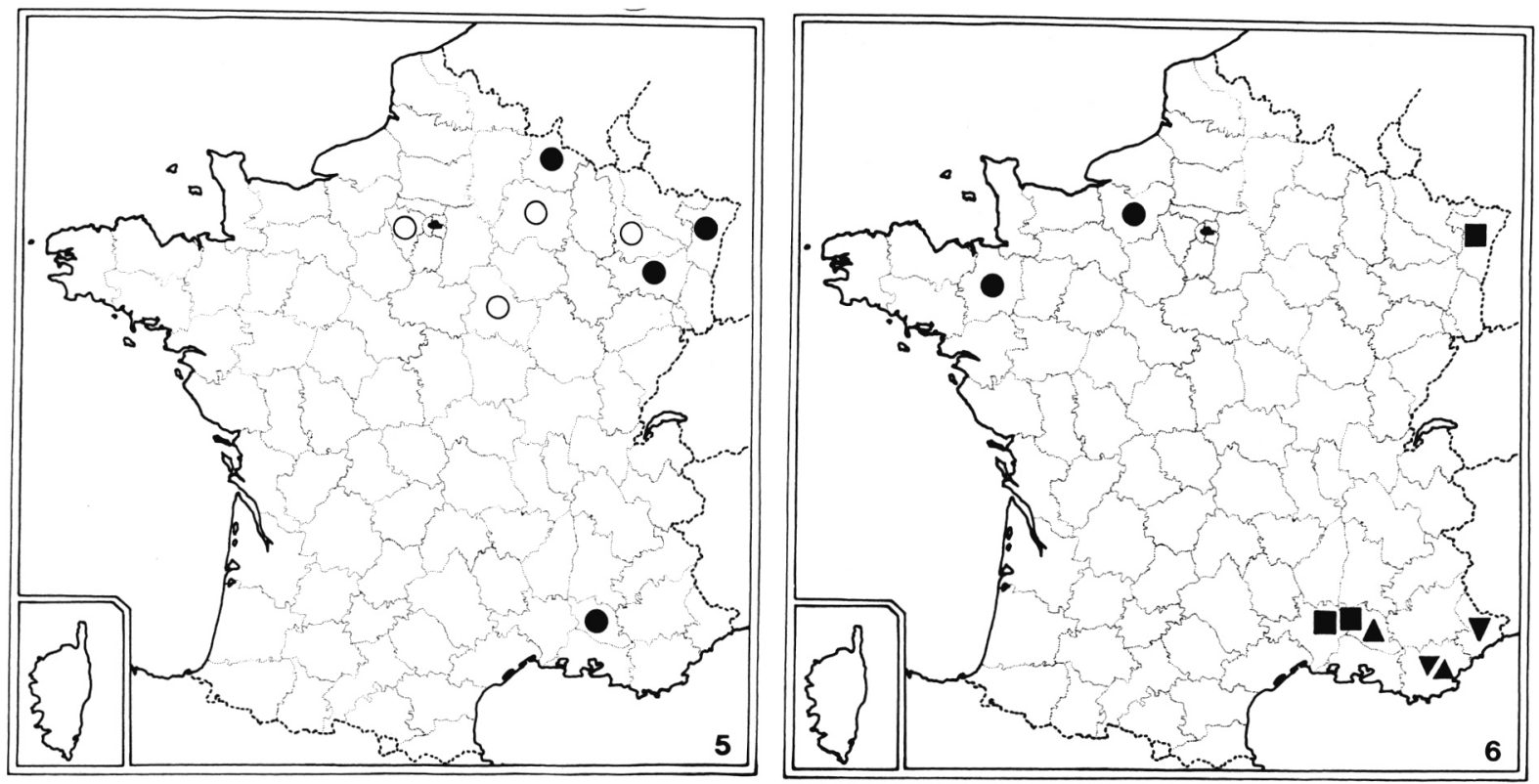

Cartes. - 5: Départements où Simulium ornatum a été signalé comme piquant l'homme (cercle noir : femelle capturée pendant la piqûre; cercle blanc: autres critères d'identification de l'insecte en cause); $6:$ Autres simulies attaquant l'homme, toutes références basées sur la capture de l'insecte pendant la piqûre (cercle : S. posticatum; triangle pointe en bas : S. variegatum; triangle pointe en haut : S. bezzii; carré : S. erythrocephalum).

- S. bezzii, limitée au Sud-Est, comme S. variegatum, du moins en tant qu'anthropophile,

$-S$. erythrocephalum, signalée dans le Bas-Rhin, très agressive dans au moins une partie du Vaucluse et récemment retrouvée dans le Gard ( $\mathrm{D}^{\mathrm{r}}$ Gilot, rec.).

Trois autres espèces restent suspectes: $S$. equinum, $S$. reptans et $S$. morsitans. Il se peut que leur agression ne soit que sporadique... ou leur détermination erronée. Il est toutefois indiscutable que $S$. equinum, pratiquement ubiquiste dans notre pays, a une solide réputation de zoophilie.

Enfin le cas de $S$. maculatum, première simulie incriminée en France (Tisserant, 1863) restera une énigme. Il est à craindre que son identification soit fausse : Grenier (1953) ne l'évoque même pas comme espèce possible en France. Toutefois il s'agit d'une simulie attaquant l'homme et évoluant dans de très grandes rivières ou des fleuves : la zone d'agression, que nous avons visitée, surplombe le Rhône. Si celui-ci est, actuellement, trop pollué particulièrement en aval de Lyon, rien ne permet d'exclure qu'il hébergeait $S$. maculatum en 1863. Rivosecchi (1978) fait pour l'Italie les mêmes réserves que nous : actuellement disparue de ce pays, cet auteur a eu toutefois la preuve, par des exemplaires en collection, que cette simulie évoluait au début du siècle dans les fleuves de Lombardie et d'Émilie. Zwick et Crosskey (1980) notent également que cette espèce, raréfiée par la pollution, fut autrefois très répandue en Europe... Qu'on ne nous fasse pas dire, toutefois, que la pollution a du bon $! . .$.
Nous souhaitons que cette note sur un problème de grande actualité, tant médicale qu'entomologique, incite nos collègues à compléter ces données. Nous sommes, semble-t-il, en train de passer de la phase de nuisance à celle de problème médical, invalidant, douloureux et pouvant présenter des complications graves.

\section{RÉFÉRENCES}

Alcock (1920) : In Brumpt E., Précis de Parasitologie (p. 1415), Masson éd., Paris, 1949, 2138 p.

Bach Cap.: In Hervé et de Lanoye, Voyages dans les glaces du pôle arctique (p. 323-330) Hachette éd., Paris, 1865.

Bazex J., Dupré, Parant : Lésions verruqueuses et granulomateuses provoquées par des piqûres de moucherons. Bull. Soc. Fr. Dermat. Syphil., 1958, 3, 310.

Beaucournu-Saguez F., Chevrier S., Dubois R., Genete M., de Lemos J. B., Mutel P., Beaucournu J. C. : Étude préliminaire d'un foyer de simuliidose à Simulium posticatum Meigen, 1838 dans le département de l'Eure. Impact médical. Méd. Mal. Infect., 1990, 20, 279-283.

Beaucournu-Saguez F., Gilot B., Chevrier S., Branquet F., Hautefort B., Beaucournu J. C. : Observation en France de Simulium erythrocephalum De Geer 1776 comme agent de simuliidose humaine. Ann. Parasitol. Hum. Comp., 1992, 67, sous presse.

Beurey J., Weber M., Mougeolle J. M. : Allergies aux simulies. Ann. Méd. Nancy et Est, 1983, 22, 55-56.

Bouchet F., 1991 : In Lavaud et coll., 1991.

Bourgeois M. : Fausse allergie cutanée verno-automnale. Les réactions aux morsures de Simulies. Rev. Fr. Allergol., 1985, 25, 151-152. 
Brumpt E. : In Discussion du Rapport de la Commission de la Lèpre (pp. 174-175). Bull. Soc. Path. Exot., 1909, 2, 166-184.

Brunhes J., Philippon B. : Note faunistique sur les simulies de France (Diptères Simuliidae). L'Entomologiste, 1966, 22, 4-14.

Callot J. : Notes faunistiques sur quelques espèces de moustiques et de nématocères piqueurs. Bull. Ass. Phil. Alsace-Lorraine, 1951, 9, 72-73.

Callot J. : Notes faunistiques sur quelques diptères piqueurs. Bull. Ass. Phil. Alsace-Lorraine, 1952, 9, 97-98.

Caron A. F. : Simulies de la région marnaise : effets pathologiques à propos de soixante observations. Thèse Doct. Méd., Reims, 1990, 103 p.

Chevrier S. : Étude d'un foyer de Simulies (Dipt., Nematocera) piqueuses de l'homme : aspects écologique et médical. DEA interactions hôtes-parasites, Univ. Paris XII, 1990, 41 p. + 13 pl.

Crosskey R. W. : The blackfly fauna of the London area (Diptera: Simuliidae). Entomologist's Gazette, 1985, 36, 55-75.

Crosskey R. W. : An annotated checklist of the world Black flies (Diptera: simuliidae) (pp. 425-520). In Black flies: Ecology, Population Management, and Annotated World List. Kim K. C. et Merritt R. E. ed., Pennsylvania State Univ. et London, 1986, $\mathrm{XV}+528 \mathrm{p}$.

Crosskey R. W. : The Natural History of Blackflies. Wiley J. et Fils ed., Chichester, etc., 1990, IX + 711 p.

Crosskey R. W. : A new checklist of the blackflies of Britain and Ireland, with geographical and type information (Diptera: Simuliidae). Entomologist's Gazette, 1991, 42, 206-217.

Davies D. M., Peterson B. V., Wood D. M. : The black flies (Diptera: Simuliidae) of Ontario. Part I. Adult identification and distribution with descriptions of six new species. Proc. Entomol. Soc. Ont., 1962, 92, 70-154.

Diaz L. A., Sampaio S. A. P., Rivitti E. A. et coll. : Endemic Pemphigus Foliaceus (Fogo Selvagem) : II. Current and Historic Epidemiologic Studies. J. Invest. Dermatol., 1989, 92, 4-12.

Forbes S. A. : On black-flies and buffalo-gnats (Simulium) as possible carriers of pellagra in Illinois. Twenty-seventh Rep. State Ent. Ill., 1912, 21-55.

Gérardin I. : Les simulies de la Suippe. Thèse Doct. Méd., Reims, $1990,86 \mathrm{p}$.

Girard M. : Les métamorphoses des insectes. Hachette et Cie éd., Paris, 1868, $359 \mathrm{p}$.

Grenier P. : Notes morphologiques et biologiques sur quelques Simulies nouvelles pour la faune française. Bull. Soc. Entom. France, 1947, 52, 66-69.

Grenier P. : Simuliidae de France et d'Afrique du Nord (Systématique, Biologie, Importance médicale). P. Lechevallier éd., Paris, 1953, $170 \mathrm{p}$.

Grouiller G., Richez F., David J. M. : Intérêt de l'héparinate de calcium injectable dans le traitement des piqûres de Simuliidae. Méditer. Méd., 1984, 318, 5-9.

Jacques A. C. : Les Simulies : à propos d'un cas d'envenimation de bétail par les simulies dans les Vosges. Thèse Doct. Pharm., Paris V, 1984, 40 p. + biblio. non paginée.

Johannsen O. A. : Simuliidae. In Aquatic insects in New York State. Bull. N. Y. State Mus., 1903, 68, 336-388.

Joubert L., Pravé M. : Rôle des simulies (Tetisimulium bezzii Corti, 1914 et Odagmia du groupe ornatum) dans les enzooties hivernales occasionnelles de myxomatose en Haute-Provence. Premiers résultats. Bull. Soc. Sci. Vét Méd. Comp., Lyon, 1972, 74, 349-351.

Kassai T., Cordero Del Campillo M., Euzeby J., Gaafar S., Hiepe Th., Himonas C. A. : Standardized Nomenclature of Animal Parasitic Diseases (SNOAPAD). Vet. Parasitol., 1988, 29, 299-326.

Lavaud F., Bouchet F., Cossart C., Caron A. F., Guérin B. : Effets pathologiques des piqûres de simulies. Étude clinique et recherche d'un terrain à risque à propos de 30 observations en région rémoise. Rev. Fr. Allergol., 1991, 31, 201-208.

Marchoux E., Bourret G. : Enquête étiologique dans un foyer de lèpre. Bull. Soc. Path. Exot., 1908, 1, 288-292.

Mégnin J. P. : La question du transport et de l'inoculation des virus par les mouches. J. Anat. Physiol., 1875, 11, $121-133+1 \mathrm{pl}$.

Mégnin P. : Les parasites articulés chez l'homme et les animaux utiles (maladies qu'ils occasionnent). Masson G. éd., Paris, 1895, 510 p. $+26 \mathrm{pl}$.

Meller-Melloul C. : Arabies : des petites bêtes qui piquent. Impact Médecin Quotidien, 1991, 64, 22.

Michon P., Lienhart R. : Lésions dermatologiques dues aux piqûres de la simulie ornée (Simulium ornatum). Rev. Méd. Nancy, 1946, 71, 101-102.

Noirtin C., Boiteux P., Beaucournu-Saguez F., Matile L., Lemasson C., Gotto B. R., Morgan W. T. : Mort de 25 animaux de ferme (dont 24 bovins) par piqûres de simulies dans les Vosges. Bull. Soc. Vét. Prat. France, 1979, 63, 3-10.

Noirtin C., Boiteux P., Guillet P., Dejoux C., BeaucournuSaguez F., Mouchet J. : Les simulies, nuisance pour le bétail dans les Vosges : les origines de leur pullulation et les méthodes de lutte. Cah. ORSTOM, Sér. Ent. méd. et Parasitol., 1981, 19, 101-102.

Peterson B. V. : The black flies of Iceland (Diptera: Simuliidae). Can. Entomol., 1977, 109, 449-472.

Phisalix M. : Animaux venimeux et venins. La fonction venimeuse chez tous les animaux; les appareils venimeux, les venins et leurs propriétés; les fonctions et usages des venins; l'envenimation et son traitement. Masson et Cie éd., Paris, 1922, 1, $\mathrm{xv}+656 \mathrm{p}$.

Pinheiro F. P., Bensabath G., Costa D., Maroja O.M., Lins Z. C., Andrade A. H. P. : Hemorrhagic syndrome of Altamira. Lancet, 1974, 639-642.

Prévoteau C. : A propos d'accidents cutanés consécutifs à des piqûres de simulies observés à l'hôpital de Reims (France). Thèse Doct. Pharm., 1990, 86 p.

Railliet A. : Traité de Zoologie médicale et agricole. Asselin et Houzeau éd., Paris, 1895, 1303 p.

Riley C. V. : Report of the entomologist. United States Department of Agriculture Report 1886, 1887, 459-592.

Rivière de, 1826 : In Rolland E.

Rivosecchi L. : Fauna d'Italia, Simuliidae Diptera Nematocera. Calderini éd., Bologna, 1978, 533 p.

Rolland E. : Faune populaire de la France, XIII : Les insectes (1 ${ }^{\mathrm{re}}$ partie), sans éd., Paris, 1911, $217 \mathrm{p}$.

Rousset J. J., Dreyfuss G., Gantier J. C. : Piqûres de simulies en France. Presse Méd., 1983, 12, 1774.

Rousset J. J., Dreyfuss G., Gantier J. C. : Piqûres de simulies. Pratique Méd., 1985, 26, 44-45.

Schönbauer J. A.: Geschichte der schädlichen Kolumbatczer Mücken im Bannat, als ein Beitrag zur naturgeschichte von Ungarn. Patzowsky éd., Vienna, 1795, $100 \mathrm{p}$.

Tisserant M. : Piqûres de moucherons. J. Méd. Vét. Lyon, 1863, 173-178.

Verdaguer M., Grillat J. P., You B. : Pseudo-allergies aux simulies et environnement boisé. Bull. Actual. Thérap., 1985, 29752979.

Williston S. W. : Manual of the families and genera of North American Diptera. J. T. Hathaway ed., New Haven, Connecticut, 1908 (in Davies et coll., 1962).

Zurcher, Margollé : Les météores. Hachette et Cie., Paris, 1864, $330 \mathrm{p}$.

Zwick H., Crosskey R. W. : The Taxonomy and Nomenclature of the Blackflies (Diptera: Simuliidae) described by J. W. Meigen. Aquatic Insects, 1980 (1981), 2, 225-247.

(C) Masson, Paris 1992 